\title{
Comparative genomic analysis reveals organization, function and evolution of ars genes in Pantoea spp.
}

\author{
L.Y. Wang \& C.Y. Jing \\ Research Center for Eco-Environmental Sciences, Chinese Academy of Sciences, Beijing, P.R. China
}

\begin{abstract}
Numerous genes are involved in various strategies to resist toxic arsenic (As). However, the As resistance strategy in genus Pantoea is poorly understood. In this study, a comparative genome analysis of 23 Pantoea genomes was conducted. Two vertical genetic ars $C$-like genes without any contribution to As resistance were found to exist in the 23 Pantoea strains. Besides the two $\operatorname{ars} C$-like genes, As resistance gene clusters arsRBC or arsRBCH were found in 15 Pantoea genomes. These ars clusters were found to be acquired by horizontal gene transfer (HGT) from sources related to Franconibacter helveticus, Serratia marcescens, and Citrobacter freundii. During the history of evolution, the ars clusters were acquired more than once in some species, and were lost in some strains, producing strains without As resistance capability. This study revealed the organization, distribution and the complex evolutionary history of As resistance genes in Pantoea spp. The insights gained in this study improved our understanding on the As resistance strategy of Pantoea spp. and its roles in the biogeochemical cycling of As.
\end{abstract}

\section{INTRODUCTION}

A series of arsenite $(\mathrm{As}(\mathrm{III}))$ oxidizing bacteria have been successfully isolated and employed to transform As(III) to arsenate (As(V)) for As remediation, because $\mathrm{As}(\mathrm{V})$ is much more strongly adsorbed than As(III). Considering the ubiquitous existence of As(III) oxidizing bacteria in groundwater, we hypothesize that the indigenous As(III) oxidizing bacteria in groundwater should affect the speciation of adsorbed As in filters.

In traditional molecular biology research, As resistance traits are revealed primarily based on the cultivation of a specific strain, and it is impossible to study the As strategy of all strains in a genus. Nevertheless, the genomic sequence of a strain contains nearly all of the genetic information. Therefore, fundamental knowledge such as the phylogenetic, the genetic traits of As resistance and its evolutionary history can be obtained through comparative genomic analysis (Colston et al., 2014).

Pantoea is a genus of Gram-negative, facultative anaerobic bacteria. Currently, the genus contains twenty-six species. In 2013, the strain Pantoea sp. IMH was an isolate that reported firstly as the strain having the As resistance capability within Pantoea species (Wu et al., 2013). Further, we sequenced the genome of Pantoea sp. IMH and found two ars clusters (arsR1B1C1H1 and arsR2B2C2H2) co-contributing to its As resistance (Wang et al., 2016). However, the evolutionary history and genetic traits of As resistance in genus Pantoea are not fully understood.

Herein, we present the first study of the genetic traits of As resistance in Pantoea spp., as well as their evolutionary history. Two vertically transmitted $\operatorname{ars} C$ like genes without any contribution to As resistance were found to exist in the 23 Pantoea strains. Besides these two ars $C$-like genes, As resistance gene clusters arsRBC or arsRBCH were found in 15 Pantoea genomes. These ars clusters were acquired by horizontal gene transfer (HGT) from sources related to Franconibacter helveticus, Serratia marcescens, and Citrobacter freundii. The insights gained in this study improve our understanding on the complex evolutionary history of As resistance genes and their roles in the biogeochemical cycling of As.

\section{METHODS/EXPERIMENTAL}

\subsection{Comparative genomics}

All of the orthologous pairs between Pantoea test genomes were identified by Pan Genome Analysis Pipeline (PGAP). The common dataset of shared genes among test strains was defined as their core genome. The total set of genes with test genomes was defined as the pan genome. The set of genes in each strain not shared with other strains was defined as the unique genes.

\subsection{Strains, plasmids and culture conditions}

E. coli and Pantoea strains were grown in LB medium (per liter contains: $10 \mathrm{~g}$ tryptone, $5 \mathrm{~g}$ yeast and $10 \mathrm{~g}$ $\mathrm{NaCl}$ ) or LB plates (LB medium with w/v $1.5 \%$ agar) at $30^{\circ} \mathrm{C}$. When appropriate, antibiotics were added at the following concentration: $100 \mu \mathrm{g} \mathrm{mL}^{-1}$ ampicillin. Resistance to As species was tested by plating serial 
dilutions of cultures of each strain onto agar plates containing filtered sodium arsenate $\left(\mathrm{Na}_{3} \mathrm{AsO}_{4}\right)$.

\subsection{Construction of the recombinant plasmids and E. coli strains}

A $3.86 \mathrm{~kb}$ BamHI-XbaI DNA fragment containing the complete ars 1 cluster of $P$. stewartii $\mathrm{S} 301$, a $3.43 \mathrm{~kb}$ Bam HI-XbaI DNA fragment containing the complete ars 2 gene cluster of $P$. agglomerans Tx10, a 860 bp BamHI-XbaI DNA fragment containing the complete arsC1-like gene of $P$. stewartii DC283 and a 942 bp BamHI-XbaI DNA fragment containing the complete arsC2-like gene of $P$. stewartii DC283 were PCR amplified with primers. The above PCR products were ligated to the BamHI- $X b a \mathrm{I}$ site of plasmid pUC18, yielding plasmids pUC18-ars1, pUC18-ars2, pUC18-arsC1-like and pUC18-arsC2-like. Then the plasmids were transferred to $E$. coli AW3110, yielding the recombinant E. coli AW3110-ars1, E. coli AW3110-ars2, E. coli AW3110-arsC1-like and E. coli AW3110-arsC2-like strains, respectively.

\section{RESULTS AND DISCUSSION}

\subsection{Distribution and organization of As-related genes in Pantoea genomes}

The ars genes in a genome are prone to group together as ars clusters (arsRBC and arsRBCH). Although comparison of the COG assignments of 23 genomes revealed that the DNA sequences between homologous genes within these ars clusters are conserved, some variations exist in DNA sequences, which can be divided into two sub-groups (ars1 and ars2). Unlike the two ars clusters in Pantoea sp. IMH, only one ars cluster, either ars 1 or ars 2 , was observed in other strains. The ars gene clusters generally exhibited more than $80 \%$ identity within each sub-group and about $54 \%$ identity between two sub-groups. Actually, the ars clusters were not detected in 8 strains including Sc1, BL1, 9140, DC283, MP7, C91, ND04 and FF5. Moreover, two arsC-like genes with only $25 \%$ homology (arsCl-like and arsC2-like) were found in the 23 genomes. Based on the different ars genes distributions, the 23 strains were categorized into 4 sub-groups.

\subsection{Evolution and the origin of ars clusters}

The distribution and organization of ars genes in Pantoea raise a question as to their evolution. We detected the $\mathrm{G}+\mathrm{C}$ content of ars clusters and their corresponding genomes. The results indicated that these ars clusters may be acquired in Pantoea strains by HGT. To further elucidate the evolution of the ars gene clusters, we compared the chromosomal regions flanking the ars gene clusters among the 23 Pantoea strains and found that the genes in the upstream and downstream regions were conserved among strains of the same species.
To gain insights into the origin of ars genes clusters in Pantoea, a NJ phylogenetic tree was constructed based on the ArsRBC protein sequences. The results imply that the ars 1 cluster may be acquired via HGT from Franconibacter helveticus, and ars 2 from Serratia marcescens and Citrobacter freundii in early evolutionary history.

\subsection{Functional analysis of ars gene and arsC-like genes}

The growth of the yielded recombinant $E$. coli strains E. coli-ars1 and E. coli-ars2, was tested in $5 \mathrm{mM}$ concentration $\mathrm{As}(\mathrm{V})$. Both $E$. coli-ars1 and E. coli-ars2 survived in $5 \mathrm{mM} \mathrm{As}(\mathrm{V})$, and $E$. coli-ars1 grew better than $E$. coli-ars2. This result suggests that both the ars 1 and $\operatorname{ars} 2$ clusters enabled E. coli AW 3110 to resist As, and ars 1 seemed to have a more effective As resistance capability than ars 2 . Neither the arsCl-like nor arsC2-like gene enables E. coli AW3110 to resist As. In line with the alignment result, the function analysis demonstrates that these two ars $C$-like genes do not contribute to As resistance.

\section{CONCLUSIONS}

Arsenic resistance gene clusters ars $R B C$ or ars $R B C H$ were found in most of Pantoea genomes. These ars clusters were found to be acquired by horizontal gene transfer (HGT) from sources related to Franconibacter helveticus, Serratia marcescens, and Citrobacter freundii. During the history of evolution, the ars clusters were acquired more than once in some species, and were lost in some strains, producing strains without As resistance capability. Our results improved the understanding of the As resistance strategy in Pantoea spp.

\section{ACKNOWLEDGEMENTS}

We acknowledge the financial support of the National Basic Research Program of China (2015CB932003), the Strategic Priority Research Program of the Chinese Academy of Sciences (XDB14020302), and the National Natural Science Foundation of China (41373123, 41425016, 41503094, and 21321004). We thank Yongguan Zhu for the strain E. coli AW3110.

\section{REFERENCES}

Colston, S. M., Fullmer, M. S. \& Beka, L. 2014. Bioinformatic genome comparisons for taxonomic and phylogenetic assignments using aeromonas as a test case. MBio. 5(6): 2150-7511.

Wang, L., Zhuang, X., Zhuang, G. \& Jing, C. 2016. Arsenic resistance strategy in Pantoea sp. IMH: organization, function and evolution of ars genes. Sci. Rep. 6, 39195.

Wu, Q., Du, J., Zhuang, G. \& Jing, C. 2013. Bacillus sp. SXB and Pantoea sp. IMH, aerobic As(V)-reducing bacteria isolated from arsenic-contaminated soil. J. App. Microbiol. 114: 713-721. 